\title{
Electrophysiological signs of arteriovenous malformations of the spinal cord
}

\author{
CARMEL ARMON, JASPER R DAUBE \\ From the Electromyography Laboratory, Department of Neurology, Mayo Clinic and Mayo Foundation, \\ Rochester, Minnesota, USA
}

SUMMARY A characteristic pattern of electrophysiological changes was found in 24 patients with confirmed spinal cord arteriovenous malformations (AVMs). The AVMs were limited to the thoracic cord in seven, involved the conus and the cauda equina in 10, and involved all levels in six. Of the patients, $88 \%$ had at least one definite electrophysiological abnormality: nerve conduction studies showed abnormal results in $43 \%$ (10 of 23), needle electromyography in $77 \%$ (17 of 22 ), and tibial somatosensory evoked potentials in $88 \%$ ( 7 of 8 ). The pattern of involvement was of scattered, multiple, bilateral thoracolumbosacral radiculopathies, consistent with axonal or neuronal destruction, associated with paraspinal fibrillations or abnormal activation of motor unit potentials. Electrophysiological abnormalities were seen in most patients with lower motor neuron clinical deficit. These abnormalities depended on the caudal extension of the AVM, on an arterial supply at $\mathrm{T}-10$ or below, and on the duration of symptoms. In addition to the expected abnormalities in the distribution of the AVM location, four patients had electrical changes at a distance, which may hav been due to venous stasis.

The present understanding of spinal cord arteriovenous malformations (AVMs) is based on the knowledge of the normal ${ }^{12}$ and abnormal ${ }^{3}$ spinal vascular anatomy. This understanding has been enhanced by the analysis of their pathology ${ }^{4}$ and by the description of the dural type of AVM. ${ }^{5}$ In particular, it has been established that dural AVMs are more common ${ }^{6}$ than those with significant intramedullary components. Recent reviews ${ }^{38-14}$ summarise the classification, pathophysiology, clinical presentation, investigation, and treatment of AVMs, but do not describe electrophysiological testing; no previous reports of such evaluations could be found in the English language literature. Preoperative testing of patients before surgical monitoring showed a significant number of abnormalities; we therefore reviewed an experience at the Mayo Clinic with spinal cord AVMs. Our purpose was twofold: first, to determine if there were characteristic electrical changes an electromyographer could recognise, and second, to determine if the pattern of abnormalities could increase our understanding of the pathophysiology and natural history of AVMs.

Correspondence to: Jasper R Daube, MD, Mayo Clinic, 200 First Street SW, Rochester, Minnesota 55905, USA.

Received 3 January 1989.

Accepted 15 April 1989

\section{Methods}

We reviewed the records of 114 patients in whom a diagnosi of spinal cord AVM was made or considered and a formaE neurological examination had taken place at the Mayo Clinic between 1977-87. Fourteen of these patients had cervical AVMs and were not electrophysiologically tested. Of the 100 patients in whom thoracolumbar AVM was considered, 65 had the diagnosis confirmed by angiography or surgery, or both. Thirty six of these 65 patients had electrophysiological studies done at the time of or before their AVMs were treated. Of these 36,12 were excluded because of the following: diabetes (3), peripheral neuropathy (3), lumbosacral root disease or other pathological conditions overlapping the distribution of the AVM (7), myelopathy at a higher level (1), or local radiation treatment (1). None of the patients showed signs of a lumbosacral plexopathy, a polyradiculopathy outside the thoracic or lumbosacral distribution, or motor neuron disease. The clinical histories, the findings on neurological examination and the results of electrical testing, myelography, angiography, and surgery in the remaining 24 patients were abstracted and compared with one another.

Electrophysiological testing was performed in 12 patients as a baseline for surgical monitoring. The other patients were tested as part of their clinical evaluation. Nerve conduction studies used standard surface stimulating and recording techniques. ${ }^{15}$ Findings on electromyography were recorded and summarised in a semiquantitative manner. Fibrillation potentials were graded 0 to $4(0$, none; $0 \cdot 5$, equivocal; 1 , 
minimal and scattered; 2 , present in all areas; 3 , moderate numbers in all areas; 4 , filling the baseline). Motor unit potential changes were also graded 0 to $4(0$, none; 0.5 , equivocal; 1 to 4 , severity of the maximal abnormality among increased duration, increased amplitude, and decreased recruitment). The poorest results were recorded for a muscle examined bilaterally. Poor or irregular activation of motor unit potentials (a sign of upper motor neuron disease) was also recorded. Tibial somatosensory evoked potentials (ankle stimulation) were recorded over the low back (L-1 to iliac crest-N22), the neck (C-5 to $\left.\mathrm{F}_{\mathrm{z}}-\mathrm{N} 30\right)$, and the scalp $\left(\mathrm{C}_{\mathrm{z}}\right.$ to $\mathrm{F}_{\mathrm{z}}-\mathrm{P} 38$ ). Electromyographic changes in individual muscles were grouped together to give an aggregate for those innervated by one nerve root and also for the entire limb by calculating the means of definite changes. For this calculation, muscles with equivocal findings were considered normal.

Discrete distributions of variables of interest were compared using the chi-squared test, with Yate's correction for 2 $\times 2$ tables. Means of subgroups were compared using Student's $t$ test (one-tailed). Correlations between continuous or quasi-continuous variables were performed by linear regression analysis. The effects of AVM location were evaluated statistically by subdivision and comparison of the patients with the AVM at T-9 or above with those whose AVM was at T-10 and below, with or without involvement of the cauda, and with those in whom the AVM extended over both cord and conus. The effect of the arterial supply was assessed by comparison of patients with a supply at vertebral level T-9 and above with those with a supply at T-10 and below. Additional analyses compared patients by the locations of the upper and lower limits and the midpoint of the AVM and by the specific level of the arterial supply. Mean values are followed by the standard error of the mean, except when standard deviation (SD) is indicated.

\section{Results}

\section{Clinical}

Nineteen men and five women took part in the study. Their ages ranged from 36 to 74 years $(58 \cdot 0$, SD 10.6). The duration of their symptoms before electrophysiological testing ranged from 3 months to 12 years $(30.8$, SD 36.8 months). The diagnosis of AVM was made between 3 months and 12 years after the onset of symptoms (33.5, SD $37 \cdot 1$ months). All the patients experienced weakness, $88 \%$ had sphincter disturbance, $79 \%$ had numbness and $71 \%$ reported pain. Symptoms progressed slowly in 23 patients and were remitting and relapsing in one. Upper motor neuron signs were present in 16 of 24 patients $(67 \%)$ and lower motor neuron weakness in 17 of $23(74 \%)$. The sensory loss was proprioceptive in 14 of 23 patients $(61 \%)$ and nociceptive in 17 of $23(74 \%)$. Rectal tone or anal reflex was abnormal in 10 of 16 patients examined $(63 \%)$. In $12(50 \%)$ of the patients, the diagnosis of AVM was made or considered by the examining clinician at the initial presentation and before electrophysiological studies. Other diagnoses considered were myelitis, demyelinating disease of the spinal cord (multiple sclerosis), spinal stenosis, polyradiculopathy, polyneuritis, peripheral neuropathy, myasthenia gravis or myopathy.

AVM was identified on myelography in 21 of the 24 patients $(88 \%)$. Among the three others, a second myelogram identified AVM in one, myelography showed an extradural mass that was found at surgery to be an AVM in one, and the negative myelogram was followed by diagnostic angiography in one; AVM was visualised on angiography in 20 of the 22 patients $(91 \%)$ in whom it was performed, one of them had had a negative myelogram. One patient with an intradural AVM on myelography did not have angiography before surgery.

The upper limits of the AVM ranged from T-3 to L-1 and the lower limits from T-6 to the cauda equina. None of the AVMs involved the cervical cord. The levels of the AVMs are listed in table 1. The source of arterial blood supply to the AVM is also shown in table 1. It ranged from as high as a T4-5 intercostal artery down to a sacral feeder from the internal iliac artery.

Nine AVMs were treated with embolisation only, 12 with surgery only and two with embolisation and surgery later; one was not treated. In 1985, embolisation became the treatment of choice, with surgery reserved for patients in whom embolisation had failed or who had repeated recurrences of the AVM after embolisation.

\section{Electrophysiological}

The results of nerve conduction studies (NCS) are shown in table 2 . Seven patients had abnormal results in the peroneal conduction studies, and seven had abnormal findings on the tibial conduction studies. One patient with a low amplitude tibial/abductor hallucis compound muscle action potential (CMAP) and an absent peroneal/extensor digitorum brevis CMAP also had a low peroneal/anterior tibial CMAP with a normal conduction velocity. The mean peroneal and tibial CMAPs and conduction velocities were lower and the mean tibial distal latency was longer than normal $(P<0.01)$. The greatest changes were in the CMAP amplitude, with more modest changes in

Table 1 Arteriovenous malformation levels in 24 patients

\begin{tabular}{llll}
\hline & \multicolumn{2}{l}{ Number of malformations } \\
\cline { 2 - 4 } Vertebral level & $\begin{array}{l}\text { Anatomic } \\
\text { location }\end{array}$ & $\begin{array}{l}\text { Arterial } \\
\text { supply }\end{array}$ \\
\hline T-9 and above & & 7 & 11 \\
T-10 and below & 7 & 16 & 11 \\
$\begin{array}{c}\text { Conus only } \\
\text { Conus and cord } \\
\begin{array}{c}\text { Conus and cauda } \\
\text { Not specified }\end{array}\end{array}$ & 6 & & \\
\hline
\end{tabular}


Table 2 Nerve conduction studies in 23 patients with spinal arteriovenous malformations

\begin{tabular}{|c|c|c|c|c|c|c|c|c|c|}
\hline \multirow[b]{2}{*}{ Nerve } & \multirow{2}{*}{$\begin{array}{l}\text { Number } \\
\text { tested }\end{array}$} & \multicolumn{2}{|c|}{ Amplitude, $m V$ or $\mu V^{*}$} & \multicolumn{2}{|c|}{ Conduction velocity, $\mathrm{m} / \mathrm{s}$} & \multicolumn{2}{|c|}{ Distal latency, $m s$} & \multicolumn{2}{|l|}{ F latency, $m s$} \\
\hline & & Mean $(S D)$ & Range & Mean $(S D)$ & Range & $\operatorname{Mean}(S D)$ & Range & Mean $(S D)$ & Range \\
\hline $\begin{array}{l}\text { Peroneal } \\
\text { Tibial } \\
\text { Sural }\end{array}$ & $\begin{array}{l}23 \\
20 \\
22\end{array}$ & $\begin{aligned} 3.6 & (2 \cdot 8) \dagger \\
6.7 & (4 \cdot 8) \dagger \\
13.2 & (7 \cdot 6)\end{aligned}$ & $\begin{array}{l}0-10.5 \\
0-18.5 \\
0-29.0\end{array}$ & $\begin{array}{l}44.1(3.5) \dagger \\
44.9(3.6) \dagger \\
49.2(6 \cdot 1)\end{array}$ & $\begin{array}{l}38 \cdot 9-51 \cdot 3 \\
39 \cdot 6-50 \cdot 0 \\
42 \cdot 0-61 \cdot 0\end{array}$ & $\begin{array}{l}4.5(1 \cdot 1) \\
4.4(0.8) \ddagger \\
3.7(0.3)\end{array}$ & $\begin{array}{l}3 \cdot 0-7 \cdot 9 \\
3 \cdot 1-6 \cdot 8 \\
3 \cdot 1-4 \cdot 2\end{array}$ & $\begin{array}{l}52 \cdot 2(10 \cdot 1) \\
52 \cdot 3 \quad(6 \cdot 3) \\
-\end{array}$ & $\begin{array}{l}44 \cdot 0-72 \cdot 0 \\
44 \cdot 0-63 \cdot 0 \\
-\end{array}$ \\
\hline
\end{tabular}

*Sural nerve.

†Significantly lower than the mean for age-matched normals $(P<0.01)$.

†Significantly longer than the mean for age-matched normals $(P<0.01)$.

the velocities and latencies. Twenty two patients had normal results of sural sensory conduction studies; one patient who did not have the sural nerve tested had normal results in the superficial peroneal and medial plantar studies.

In seven of the eight patients who had tibial somatosensory evoked potentials (SEPs) recorded, the P38 (scalp) response was present, its amplitude ranging from 0.3 to $2 \mu \mathrm{V}$. Its mean was $0.8 \mu \mathrm{V}$ (SD 0.22 ), significantly lower than the normal of $2.4 \mu \mathrm{V}(P<$ 0.01 ). The mean P38 latency (range, $42-53 \mathrm{~ms}$ ) was $48.4 \mathrm{~ms}$ (SD 1.58), significantly longer than the normal of $38.0 \mathrm{~ms}(P<0.01)$. Seven of eight patients $(88 \%)$ had either an abnormal P38 amplitude or latency. The N22 (lumbar) responses were present with either unilateral or bilateral tibial stimulation in only three of eight patients and all of them had normal or borderline latencies (23.2-28.4 ms). In these three patients with N22 responses, the N22-P38 interpeak latency was prolonged at $20 \cdot 2-23.8 \mathrm{~ms}$ (normal, 12.2-20.0 ms); absent $\mathrm{N} 30$ (cervical) responses prevented further localisation of the slowing.

Electromyography (EMG) was performed in 22 patients. Seven patients had poor activation, one had "irregular" activation, and the remaining 14 had normal activation. Table 3 summarises the abnormalities on EMG, according to root level. For each level, motor unit potential changes were more prevalent than fibrillation potentials and the prevalence of both fibrillation potentials and motor unit potential changes was greater in the distal than in the proximal muscles. Abnormal findings were least prevalent in the paraspinal muscles.

Comparisons of frequency of abnormalities on the electrophysiological studies can be summarised as follows: SEPs were abnormal in seven of eight patients tested $(88 \%)$ and were the only abnormality in one patient. EMG findings were clearly abnormal in 17 of 22 patients tested $(77 \%)$ and were the only abnormality in eight. In four additional patients, minimal paraspinal irritability was the only EMG abnormality, and in two of the four, it was the only electrophysiological abnormality. NCS results were abnormal in 10 of the 23 patients tested $(43 \%)$ and were always associated with other electrophysiological abnormalities.

Among the 17 patients who had definite EMG abnormalities, there were nine in whom the diagnosis of AVM was neither known nor considered by the referring physician. However, the abnormalities were milder in those in whom the diagnosis was not made, mean total limb fibrillation potentials were $0.76 \mathrm{SD}$ ? 0.22 compared with 1.57 SD 0.36 , and motor unit potential changes were $1 \cdot 12$ SD 0.21 compared with $2 \cdot 12$ SD 0.43 . In summary, 21 of $24(88 \%)$ patients had at least one definite electrophysiological abnormality. In two other patients, the only abnormality was minimal paraspinal irritability. Only one patient with spinal AVM below T-1 had no abnormal electrophysiological findings.

Table 3 Extent of abnormalities on needle electromyography in 22 patients with spinal arteriovenous malformations

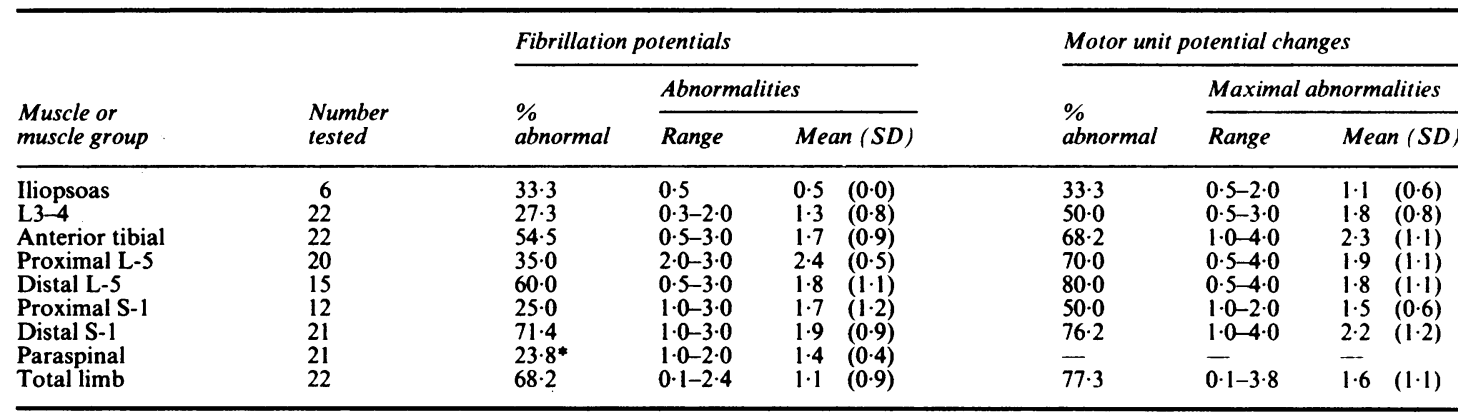

*Four additional patients $(19 \%)$ had only minimal irritability in a single paraspinal muscle. 


\section{Correlations}

Comparisons were made among the variables measured to help identify the significance of the electrophysiological changes. As expected, the location of the AVM was related to the clinical findings. Lower motor neuron weakness was present in all 10 AVMs involving only the conus, in two of six that involved only the cord and in five of six that involved both the cord and the conus. The mean lower extent of AVMs in patients with lower motor neuron weakness was T-11.2 (SD 0.49) (14 patients) and was significantly lower $(P<0.01)$ than that of those without lower motor neuron weakness, T-8.67 (SD 0.76) (six patients). Similar correlations with lower motor neuron weakness were found for the midpoint of the AVM but not for its upper limit. Pain, upper motor neuron weakness, abnormal rectal findings, and proprioceptive or nociceptive impairment were unrelated to the location of the AVM. Upper motor neuron weakness was found in five of seven patients with AVMs involving only the cord, in five of six with AVMs involving both cord and conus and in five of 10 with AVMs involving only the conus and cauda.

The level of the blood supply to the AVM showed correlations similar to those of the level of the AVM itself. The mean level of arterial supply was lower in 16 patients with lower motor neuron weakness than in six without (T-10.6, SD 0.95, compared with T-7.8, SD $1.2, P<0.1)$ and was also lower in 13 patients with proprioceptive impairment than in eight without (T-11.4, SD 1.1, compared with T-7.7, SD $0 \cdot 88, P<$ 0.025 ).

Unexpectedly, both lower motor neuron weakness and the level of AVM were found to be correlated with the time to diagnosis. Lower motor neuron weakness in 17 patients correlated with a shorter time to diagnosis $(20 \cdot 8$, SD $3 \cdot 3$, compared with $53 \cdot 3$, SD $21 \cdot 4$, months; $P<0.025)$ and with a shorter time to presentation $(20 \cdot 5$, SD $3 \cdot 4$, compared with $41 \cdot 7$, SD 22.5 , months; $P<0 \cdot 1)$. The lower the AVM extended to involve the conus, the shorter these time intervals were. When the vertebral level of the lower limit of the AVM was used as a co-ordinate, the correlation of time to diagnosis to the lower limit of the AVM in 21 patients was $-0.61(P<0.005)$ and that of the time to presentation was $-0.49(P<0.025)$.

Significant correlations were noted between the electrophysiological findings and the level of the AVM. As the AVM extended further down, the peroneal CMAP was lower $(r=-0.51, P<0.025$, $n=20)$ and there were more motor unit potential (MUP) changes $(r=0.41, P<0.05, n=19)$.

It is important to note that despite these correlations, NCS or EMG abnormalities were present in four of the seven AVMs that were limited to the thoracic cord; they were not present in AVMs that did not extend below the T-6 vertebra. As might be expected, among the AVMs that involved the conus, a lower extension of the AVM was associated with a lower tibial CMAP $(r=-0.51, P<0.05, n=12)$ and with more MUP changes in distal L-5-innervated muscles $(r=0.53, P<0.1, n=10)$.

Tibial SEP findings did not correlate with the level of the AVM but did correlate with the level of arterial supply. The tibial N22 (lumbar) response was present with unilateral stimulation and with normal amplitude and latency in three patients with arterial supply at $\mathrm{T}-7, \mathrm{~T}-10$, and T11-12; it was absent with unilateral stimulation and present with bilateral stimulation, but with prolonged latency, in one patient with arterial supply at L-1; it was absent even with bilateral stimulation in three patients with arterial supply at L-2, at L-3 and from a sacral feeder. One additional patient with arterial supply at L-1 had an absent N22 response on unilateral stimulation but did not have bilateral SEPs performed. The P38 (scalp) latency was abnormal in four of six patients with arterial supply at $\mathrm{T}-10$ or below and in one patient with arterial supply at T-7.

Electrophysiological findings also showed correlations with some of the clinical data. Five of six patients with proprioceptive loss who had tibial SEPs performed had prolonged P38 latencies. An additional patient without proprioceptive loss had a normal P38 response. The mean tibial CMAP, but not the peroneal CMAP, was lower in patients with abnormal rectal findings. In patients with AVMs involving only the conus and cauda equina, a longer duration of symptoms was associated with a lower peroneal CMAP $(r=-0.57, P<0 \cdot 1, n=9)$ and a lower tibial CMAP $(r=-0.60, P<0.05, n=9)$. No other significant electrophysiological and clinical correlations were found, but there were correlations among some of the electrophysiological variables themselves, such as between tibial and peroneal CMAPs and between MUP changes in L-5 innervated muscles and peroneal CMAP.

Four patients who had upper motor neuron findings on clinical examination with no signs of lower motor neuron disease had clear abnormalities on EMG and NCS. In these patients, the electrical testing suggested unsuspected damage and additional diagnoses. Eight of nine other patients with both upper and lower motor neuron findings and all six patients with only lower motor neuron findings also had abnormalities on EMG and NCS.

\section{Discussion}

Clinical

The age and sex distribution and clinical findings in 
the 24 patients in this study are similar to those in previous reports. ${ }^{36}$ Patients with acute presentations were not represented in our series, most likely because electrophysiological studies were not requested. Changes in terminology and classification of spinal cord AVMs make comparisons between the series difficult. Most of the patients in this study would be considered to have a "dural" rather than an "intramedullary" AVM. ${ }^{6713}$

The electrophysiological findings in this fairly typical series of patients with AVM were much more prominent than expected or previously reported. We found motor neuronopathy at multiple lumbosacral levels in 21 of the 24 patients ( $88 \%$ ) and this provided the only evidence of lower motor neuron involvement in four of the patients. NCS were abnormal in $43 \%$, needle EMGs in $77 \%$ and tibial SEPs in $88 \%$. NCS showed the reduction in CMAP amplitudes with little change in conduction velocities, typical of a neuronopathy. The distribution of EMG changes was that of scattered, bilateral, multiple radiculopathies. The greater prevalence of abnormalities in distal than in proximal muscles and the predominance of MUP changes over fibrillation potentials are typical of chronic disorders. The abnormal activation of MUPs seen in one third of the cases suggested additional upper motor neuron disease. Normal peripheral sensory studies in $100 \%$ of patients tested and abnormal tibial SEPs in $88 \%$ of those tested are consistent with involvement at the spinal cord level. The most prevalent abnormality was a delayed or reduced scalp response; in three patients with an abnormal scalp response in whom a lumbar response could be produced, slowing of conduction was localised to a site between the lumbar and the scalp levels. The lumbar response was absent or delayed in AVMs with a low level of arterial supply.

These findings are similar to those of Koenig et al $^{16}$ showing that spinal AVMs have a consistent pattern of electrophysiological abnormalities that can alert the electromyographer to an intraspinal disorder. To examine this hypothesis, we reviewed the records of 26 additional patients suspected of having spinal AVM who did not have the diagnosis confirmed by angiography or surgery. The electrophysiological changes characteristic of spinal AVM were present in 16 of 22 patients with myelographic findings of AVM; these changes were not seen in the four patients without signs of AVM on myelography. A comparison with the electrophysiological findings caused by other intraspinal processes would be required to determine the specificity of these findings. It is possible that an intraspinal glioma or multiple sclerosis at T-9 with secondary loss of trophic factors from upper to lower motor neurons might produce the same EMG changes. However, the correlation of the changes with the level of vascular supply to the AVM and their presence in patients without upper motor neuron signs make loss of trophic factors a less likely cause.

The combination of upper motor neuron findings and sensory findings clinically and lower motor neuron findings on electrophysiological studies, although not specific, should strongly suggest AVM. This pattern was seen in four of seven patients with only upper motor neuron signs on clinical examination and in the patients with negative myelograms. Electrophysiological testing should therefore be considered in patients suspected of having lower thoracic and lumbar cord disease, even if there are few or no lower motor neuron findings. In an AVM already seen on myelography, tibial SEP can be of help, since the absence of the N22 response suggests that the arterial supply to the AVM is at the lumbar level or lower.

The correlations between the electrophysiological studies, the location of the AVM, and its time to presentation and diagnosis suggest pathophysiological mechanisms by which AVMs may produce deficits. A lower extension of the AVM was associated with a shorter course, with lower motor neuron findings at presentation, and with more electrophysiological abnormalities, indicating direct damage to the cord at the level of the AVM. In addition, ford seven patients the AVM did not extend below the T-1 1 cord level and there was definite EMG evidence of conus involvement (all radicular arteries were injected on angiography in a search for all possible sources of arterial supply). These findings demonstrate that AVMs can produce lower motor neuron abnormale ities at a distance from their anatomic location and are consistent with the current view that venous hypertension leads to cord ischaemia that extends beyond the site of the AVM.

In summary, spinal AVMs have a consistent pattern of electrophysiological abnormalities that suggests the diagnosis but may not be specific for it. Electrophysiological studies therefore may be helpful in patients with upper motor neuron findings which can be localised to the lower thoracic or lumbar cord or with lower motor neuron findings and negative myelograms. The electrophysiological results support the hypothesis that AVMs produce their findings locally and at a distance from their anatomic location by increasing the spinal venous pressure and producing venostatic cord ischaemia with a secondary motor neuronopathy and interference with sensory transmission at the dorsal root or the dorsal horn.

This article was presented in part at the meeting of the American Association of Electromyography and Electrodiagnosis, San Antonio, Texas, 15-18 October, 1987. 


\section{References}

1 Turnbull IM. Blood supply of the spinal cord. In: Vinken PJ, Bruyn GW, eds. Handbook of clinical neurology, vol 12, pt 2. Amsterdam: North-Holland, 1972:478-91.

2 Gillilan LA. Veins of the spinal cord: anatomic details; suggested clinical applications. Neurology (Minneap) 1970;20:860-8.

3 Lazorthes G. Pathology, classification and clinical aspects of vascular diseases of the spinal cord. In: Vinken PJ, Bruyn GW, eds. Handbook of clinical neurology, vol 12, pt 2. Amsterdam: North-Holland, 1972:492-506.

4 Aminoff MJ, Barnard RO, Logue V. The pathophysiology of spinal vascular malformations. J Neurol Sci 1974;23:255-63.

5 Kendall BE, Logue V. Spinal epidural angiomatous malformations draining into intrathecal veins. Neuroradiology 1977;13:181-9.

6 Symon L, Kuyama H, Kendall B. Dural arteriovenous malformations of the spine: clinical features and surgical results in 55 cases. $J$ Neurosurg 1984;60:238-47.

7 Cogen P, Stein BM. Spinal cord arteriovenous malformations with significant intramedullary components. $J$ Neurosurg 1983;59:471-8.

8 Aminoff MJ, Logue V. Clinical features of spinal vascular malformations. Brain 1974;97:197-210.
9 Aminoff MJ, Logue V. The prognosis of patients with spinal vascular malformations. Brain 1974;97:211-8.

10 Tobin WD, Layton DD Jr. The diagnosis and natural history of spinal cord arteriovenous malformations. Mayo Clin Proc 1976;51:637-46.

11 Malis LI. Arteriovenous malformations of the spinal cord. In: Youmans JR, ed. Neurological surgery: $a$ comprehensive reference guide to the diagnosis and management of neurosurgical problems, vol 3, 2nd ed. Philadelphia: WB Saunders, 1982:1850-74.

12 Oldfield EH, Di Chiro G, Quindlen EA, Rieth KG, Doppman JL. Successful treatment of a group of spinal cord arteriovenous malformations by interruption of dural fistula. J Neurosurg 1983;59:1019-30.

13 Yaşargil MG, Symon L, Teddy PJ. Arteriovenous malformations of the spinal cord. Adv Tech Stand Neurosurg 1984;11:61-102.

14 Rosenblum B, Oldfield EH, Doppman JL, Di Chiro G. Spinal arteriovenous malformations: a comparison of dural arteriovenous fistulas and intradural AVM's in 81 patients. $J$ Neurosurg 1987;67:795-802.

15 Daube JR. Nerve conduction studies. In: Aminoff MJ, ed. Electrodiagnosis in clinical neurology. 2nd ed. New York: Churchill Livingstone, 1986:265-306.

16 Koenig E, Dichgans J, Schrader V, Thron A. Neurophysiological findings in patients with Foix-Alajouanine's disease (abstract). Electroencephalogr Clin Neurophysiol 1987;66:S55. 